\title{
A urine-based biomarker for chronic prostatitis/chronic pelvic pain syndrome: a retrospective multi-center study
}

\author{
Weining Liang ${ }^{1}$, Zhigang $\mathrm{Wu}^{1}$, Guowei Zhang ${ }^{2}$, Weikang Chen ${ }^{3}$, Xiangnong Hu ${ }^{2}$, Jianjun Yang ${ }^{2}$, \\ Jie Meng ${ }^{4}$, Yan Zeng ${ }^{5}$, Hongjun $\mathrm{Li}^{6}$, Xuejun Shang ${ }^{1}$ \\ ${ }^{1}$ Department of Andrology, Jinling Hospital, The First School of Clinical Medicine, Southern Medical University, Nanjing, China; ${ }^{2}$ Department of \\ Urology, Suqian First Hospital, Suqian, China; ${ }^{3}$ Department of Andrology, the First Affiliated Hospital of Wenzhou Medical University, Wenzhou, \\ China; ${ }^{4}$ Onco Biomedical Technology (Suzhou) CO. LTD, Taicang, China; ${ }^{5}$ Department of Immunology, Jinan Military General Hospital, Jinan, \\ China; ${ }^{6}$ Urological Department of Peking Union Medical College Hospital (PUMCH), Peking Union Medical College, Chinese Academy of \\ Medical Sciences, Beijing, China \\ Contributions: (I) Conception and design: X Shang, H Li, Y Zeng; (II) Administrative support: X Shang; (III) Provision of study materials or patients: \\ X Shang; (IV) Collection and assembly of data: Z Wu, X Hu, J Yang; (V) Data analysis and interpretation: W Liang, X Hu, J Yang; (VI) Manuscript \\ writing: All authors; (VII) Final approval of manuscript: All authors. \\ Correspondence to: Xuejun Shang. Department of Andrology, Jinling Hospital, The First School of Clinical Medicine, Southern Medical University, \\ Nanjing, China. Email: shangxj98@163.com; Hongjun Li. Urological Department of Peking Union Medical College Hospital (PUMCH), Peking \\ Union Medical College, Chinese Academy of Medical Sciences, Beijing, China. Email: lihongjun@pumch.cn.
}

Background: Chronic prostatitis (CP) or chronic pelvic pain syndrome (CPPS) is one of the most common diseases in young and middle-aged men, accounting for 30\% of outpatient men in urology OPD. There are no definitive diagnostic criteria for CP or CPPS and no accepted therapies that cure the disease.

Methods: We identified 372 patients with CP diagnosed from 2015 to 2018 and collect the information of age, routine urinary test, express prostatic secretion (EPS), and NIH-Chronic Prostatitis Symptom Index (NIH-CPSI).

Results: Our study proved a correlation between the increase of prostatic exosomal proteins (PSEPs) level and NIH-CPSI scores. Spearman's correlation coefficient showed a significant level correlation between NIH-CPSI and PSEP level ( $r s=0.194, \mathrm{P}=0.0035$ ). In the meantime, the correlation was found between the PSEP level and EPS-white blood cells. Spearman's correlation coefficient showed that there was a significant hierarchical correlation between EPS-white blood cells and PSEP level ( $\mathrm{rs}=0.183, \mathrm{P}=0.001$ ).

Conclusions: These findings highlight the potential of PSEP is a practical indicator of the symptomatic progression of CP/CPPS. Applications of PSEP assay may guide drug discovery and lead to better treatment to improve the patient's quality of life.All in all, PSEP detection in urine is safe and effective, and it is worthy of further promotion and application in clinical practice.

Keywords: Chronic prostatitis (CP); prostate exosomal protein; chronic pelvic pain syndrome (CPPS); urinebased biomarker

Submitted Aug 14, 2020. Accepted for publication Oct 12, 2020.

doi: $10.21037 /$ tau-20-1268

View this article at: http://dx.doi.org/10.21037/tau-20-1268

^ ORCID: 0000-0002-5499-8633. 


\section{Introduction}

Chronic prostatitis (CP) or chronic pelvic pain syndrome (CPPS) is one of the most common diseases in young and middle-aged men and accounts for up to $30 \%$ of the outpatient male observed in the urological clinics (1). From the epidemiological survey, $4.5-10 \%$ of the male population presents symptoms of prostatitis worldwide, and $50 \%$ of men have prostatitis at some points during their lifetime $(2,3)$. Thus, CP/CPPS is of paramount importance as a medical problem for health care internationally. However, despite the intense research in the past decades, the etiology and pathogenesis of CP/CPPS are still unclear. Also, the clinical manifestation of CP/CPPS lacks specificity, making clinical diagnosis and treatment challenging $(4,5)$. The cause of CPPS has not yet been clarified, but the basic viewpoint is: CP/CPPS is a complex disease involving physical and mental factors. Even if there are obvious physical lesions that can cause pelvic pain, psychological and social factors cannot be ignored. Treatment requires the use of a multidisciplinary and comprehensive approach, including surgery, drugs, physiotherapy, psychotherapy, etc. (6). The purpose of treatment is to relieve pain, improve function and eliminate psychological barriers. Many biomarkers have also been confirmed to be involved in inflammation (7). So the research of biomarkers is not only helpful for the accurate diagnosis of CP/CPPS, but also for the development of new targeted drugs for CP/CPPS, which is important for the individualized and precise treatment of CP/CPPS.

The diagnosis of CP/CPPS has included a combined process of recording clinical symptoms and signs, routine urine tests, or culture and the express prostatic secretion (EPS), which can be retrieved by performing a rectal exam with a massage on the prostate (8). However, this is a clinical process that requires a qualified doctor to operate and often disturb the patients. Also, the EPS index may exclude other potential pelvic pain associated with urological disorders. There is a lack of effective tools for the evaluation of the disease and the judgment of effect. At present, the NIHChronic Prostatitis Symptom Index (NIH-CPSI) is a questionnaire most commonly used internationally. It was developed by experts organized by the National Institutes of Health. Most practice clinics and hospitals in our country (for example, the Jinling hospital in Nanjing; the Taicang People's Hospital and Military General Hospital in Jinan) carry out the National Institutes of Health Chronic Prostatitis Symptom Index (NIH-CPSI) to document the patient symptoms and responses to diagnose CP/CPPS. In this process, the patient must answer several questions. The doctor should calculate the NIH-CPSI score according to medical history and clinical symptoms. Therefore, it is imperative to find and introduce a valid tool of CP/CPPS surrogate for diagnosis.

Also, studies have shown increased risks of prostate cancer (PCa) for men with a history of prostatitis compared with the case-control. For example, Tomas et al. found the atypical hyperplasia in epithelial cells with dark, swelling, and prominent nucleoli in the tissue slide showing a lesion of inflammatory atrophy. Inflammatory atrophy can supply a favorable breeding ground for PCa development (9).

Exosomes are small, membrane-bound storage vesicles that mediate transport of a cytosolic cargo between the cells and to the extracellular space (10). Exosomes are produced in many cell types, including the prostate epithelial cells, where they are termed prostasomes (11). They can also be excreted to the interstitial tissue compartments when infiltrating leukocytes accumulate in response to inflammation. Thus, prostasomes can be found in seminal plasma, and urine (12). Prostasomes have been reported to elicit antioxidant effects, antibacterial activity, and immunomodulation $(13,14)$. It has been proposed prostasomes may reduce the production of reactive oxygen species (ROS) (15). Studies also suggested prostasomes inhibit the NADPH oxidase activity of polymorph nuclear neutrophils by lipid transfer from prostasomes to the plasma membrane of these cells (16). The molecular composition of human prostasomes is varied and comprises hundreds of known and unknown proteins. Prostate diseases, including prostate cancer, benign prostatic hyperplasia $(\mathrm{BPH})$, and prostatitis, present unique phenotypes at the level of their respective proteasomal proteomes (17).

Recently, antibodies against human prostasomes were generated and found to be reactive to urine samples of CP/ CPPS patients. The proteins that are immune reactive to the antibodies are appointed as prostatic exosomal proteins (PSEPs) $(18,19)$.

A multi-center clinical trial performed in China showed that CP/CPPS patients present elevated PSEP in the void urine when compared to the healthy men (20). Subsequent applications of the PSEP test confirmed the utility in many clinics across China; however, these applications have not addressed the relationship between PSEP test and current methods of diagnosing CP/CPPS. In this study, we intended to be the first to reveal the potential relationship between PSEP in urine samples and EPS indexes, including white blood cells (WBC) and lecithin corpuscles and NIH-CPSI. Our studies highlight the potential value of PSEP as an 
indicator for CP/CPPS symptoms and disease progression.

We present the following article in accordance with the MDAR reporting checklist (available at http://dx.doi. org/10.21037/tau-20-1268).

\section{Methods}

\section{Subjects}

From Sep 2015 to May 2018, 372 patients (age ranging from 20 to 61 years old, male) were recruited and diagnosed as having CP/CPPS at the Jinling Hospital Affiliated to the Nanjing University School of Medicine, the Nanjing University of Chinese Medicine Affiliated Integrated Traditional and Western Medicine, Jinan Military General Hospital. Of the 372 patients, 225 underwent an NIH-CPSI questionnaire survey (21). For inclusion into this study, CP/CPPS patients must meet the following criteria: males with CP/CPPS history and clinical symptoms, including urinary frequency, urgency, and retention, the inflammatory reaction, or reflective (perineal pain, abdominal bulge, and discomfort). A portion of patients presented with premature ejaculation or other symptoms includes infertility. Upon rectal exam, CP/CPPS patients confirmed changes of EPS found over the average person, including $\mathrm{WBC}$ and lecithin corpuscle (phosphatidylcholine) in secretion. Also, routine urinary tests or culture showed no significant anomaly of acute inflammatory cell types or other urinary tract infections. An NIH-CPSI questionnaire survey was used to report pain, symptoms of abdominal discomfort, finding urination symptoms, and quality of life to give rise to a total score.

The Institutional Research Ethics Committee approved all research analysis. The written informed consent was retrieved from all individuals, and case-report-forms (CRF) were administered during outpatient visits to collect the information of age, routine urinary test, EPS and NIHCPSI, etc. All procedures performed in this study involving human participants were in accordance with the Declaration of Helsinki (as revised in 2013).

\section{Sample collection}

Midsegment urine samples were attained in the morning and were at once frozen. They were stored at $-20^{\circ} \mathrm{C}$ until ready for use. Subjects were excluded when there were incomplete clinical data, inadequate quantities of urine samples, or grossly bloody and thick urine, or with alcohol consumption.

\section{PSEP assay}

The double-blinded PSEP-ELISA assay was performed as described according to the manufacturer's instruction [Onco Biomedical Technology (Suzhou) CO., Ltd] (20). Void human urine samples were added to the 96-well microplate trays and incubated at $37^{\circ} \mathrm{C}$ for one hour. After antibody incubation, the reaction was visualized by the addition of chromogenic tetramethylbenzidine (TMB). The resulting color development shows the amount of PSEP in urine samples. The absorbance of the samples was read at $450 \mathrm{~nm} / 630 \mathrm{~nm}$.

\section{Statistical analysis}

The statistical analysis was performed blindly. For the crosssectional study analysis, a database collected all information from each patient, including age, routine urinary test, EPS, including WBC, and lecithin corpuscle in secretion and NIH-CPSI. WBC and lecithin corpuscle stratified data in secretion and NIH-CPSI, respectively, according to different classification methods. The mean of PSEP concentration and detection rate of PSEP are calculated, respectively. Contingency tables and Spearman's correlation coefficient were used to test for independence between PSEP positive/negative status and concentration with Chisquare test statistics by SAS9.4 (The SAS software was developed by The State University of North Carolina, the USA in 1966) for each factor including WBC and lecithin corpuscle and NIHCPSI. Data were stratified by counting methods to minimize potential confounding factors when testing for association between PSEP and CP/CPPS status. The differences were significant when $\mathrm{P}<0.05$. We conduct power analyses for the assessment of our sample size by $G$ power software.

\section{Results}

The relationship between urine PSEP level and EPS-WBC number with "+/-" as an indicator of disease severity

All 372 patients were documented with EPS-WBC numbers in their case report forms (CRFs). This method stratified them from documenting WBCs, as shown in Table 1.

They were divided into distinct groups according to their WBC number in EPS. WBC number less than 9 
Table 1 Relationship between urine PSEP level and WBC numbers in EPS

\begin{tabular}{lccccc}
\hline WBC grade & Case number & PSEP positive & PSEP negative & Positive rate $(\%)$ & Mean $(\bar{x} \pm$ s) \\
\hline WBC \pm & 58 & 31 & 27 & 53.4 & $2.56 \pm 2.62$ \\
WBC+ & 116 & 76 & 40 & 65.5 & $3.23 \pm 3.29$ \\
WBC++ & 75 & 51 & 24 & 68.0 & $3.17 \pm 2.78$ \\
WBC+++ & 72 & 55 & 17 & 76.4 & $8.63 \pm 3.91$ \\
WBC++++ & 51 & 42 & 9 & 82.4 & $4.08 \pm 2.78$ \\
\hline
\end{tabular}

Chi-Square test $\chi^{2}=13.200, P=0.010$. Spearman's correlation coefficient analysis $r s=0.183, P<0.001$. WBC \pm : WBC number less than $9 / H P$; WBC+: WBC number 10-20/HP; WBC++: WBC number 21-30/HP; WBC+++: WBC number 31-40/HP; WBC+++: WBC number >40/HP. $\mathrm{P}<0.05$ is statistically significant. PSEP, prostatic exosomal protein; WBC, white blood cells; EPS, express prostatic secretion.

Table 2 The relationship between urine PSEP level and the density of EPS-lecithin corpuscles

\begin{tabular}{lccccc}
\hline Lecithin grade & Case number & PSEP positive & PSEP negative & Positive rate $(\%)$ & Mean $(\bar{x} \pm s)$ \\
\hline+ & 89 & 61 & 28 & 68.5 & $3.37 \pm 3.08$ \\
++ & 99 & 68 & 31 & 68.7 & $3.55 \pm 3.38$ \\
+++ & 117 & 80 & 37 & 68.4 & $3.74 \pm 3.48$ \\
++++ & 67 & 46 & 21 & 68.7 & $3.18 \pm 2.69$ \\
\hline
\end{tabular}

Chi-Square test $\chi^{2}=0.003, P=0.999$. Spearman's correlation coefficient analysis $r s<0.001, P=0.994$. PSEP, prostatic exosomal protein; EPS, express prostatic secretion.

under the high-power microscope is considered negative or set as \pm ; WBC number 10-20 is set as + ; WBC number $21-30$ is set as ++; WBC number $31-40$ is set as+++; WBC number $>40$ is set as ++++ . As is shown in Table 1 , with the increase of EPS-WBC number, the positive rate of PSEP showed a trend of increase. The mean PSEP concentration appeared to increase. PSEP concentration in urine sample change significantly when we analyze the dataset with the contingency table chi-square test $\left(\chi^{2}=13.200, \mathrm{P}=0.01\right)$. Spearman's correlation coefficient showed a significant rank correlation between EPS-WBC and PSEP concentration either ( $\mathrm{rs}=0.183, \mathrm{P}=0.001$ ). These data suggested that, in the current cohort of 372 patients, there was a statistically significant correlation between the number of WBC and the concentration of PSEP in the urine of CP/CPPS patients.

\section{Relationship between urine PSEP level and EPS-lecithin corpuscles}

Although the vitality EPS examination has been questioned, EPS is still widely used in the clinic because there is no ideal specific diagnostic marker. We, therefore, examined EPS-lecithin corpuscles for all patients. All 372 patients had records of the EPS-lecithin corpuscle in their CRFs. The grade of EPS-lecithin corpuscle density stratified them, as shown in Table 2. In normal EPS, a full field of lecithin corpuscles was observed with a high-power microscope, which was appointed as ++++. The density of EPS-lecithin corpuscles lower than $50 \%(++)$ per vision field under the high-power microscope is considered a sign of CP/CPPS in urological clinics. From Table 2, the data showed there was no statistical significance $\left(\chi^{2}=0.003, P=0.999\right)$ between the density of lecithin corpuscles and PSEP concentration in the urine of CP/CPPS patients when we analyzed them with contingency tables chi-square test. Also, the Spearman's correlation coefficient showed no significant rank correlation between the two either ( $\mathrm{rs}=0.001, \mathrm{P}=0.994)$.

\section{Relationship between urine PSEP level and NIHCPSI}

The CP symptom index developed by the NIH of the United States (NIHCPSI) is an established scoring method to record the symptoms of the patients. According to the severity of symptoms, NIH-CPSI is divided into mild (1-14 points), moderate (15-29 points), or severe (30-43 points) (21). Increases of NIH-CPSI are used as the indication that $\mathrm{CP} / \mathrm{CPPS}$ becomes more pronounced with more severe symptoms. 
Table 3 The relationship between the urine PSEP level and the NIH-CPSI

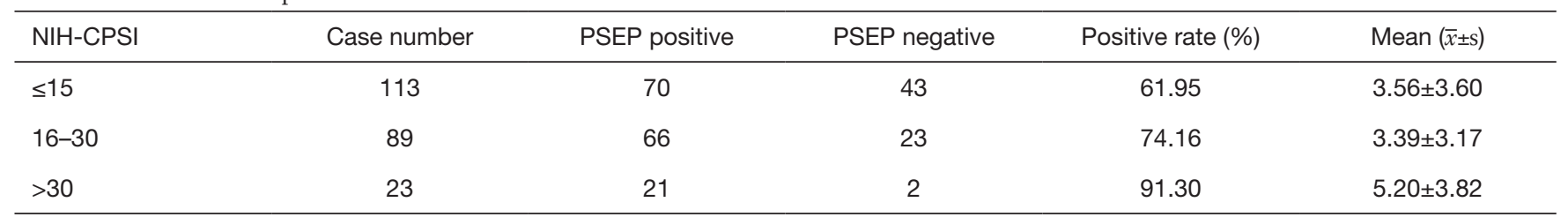

Chi-Square test $\chi^{2}=9.149, \mathrm{P}=0.0091$. Spearman's correlation coefficient analysis $\mathrm{rs}=0.194, \mathrm{P}=0.0035$. PSEP, prostatic exosomal protein; $\mathrm{NIH}-\mathrm{CPSI}, \mathrm{NIH}-\mathrm{Chronic}$ Prostatitis Symptom Index.

From the $372 \mathrm{CP} / \mathrm{CPPS}$ patients, 225 patients had NIH-CPSI records. The correlation between urine PSEP level in urine and NIH-CPSI was examined. As shown in Table 3, the rising NIH-CPSI was correlated with the increase of patients with a positive rate of PSEP. We analyzed them with the contingency table chi-square test $\left(\chi^{2}=9.149, \mathrm{P}=0.0091\right)$. Spearman's correlation coefficient showed a significant rank correlation between NIH-CPSI and PSEP concentration ( $\mathrm{rs}=0.194, \mathrm{P}=0.0035)$. Although the correlation between NIH-CPSI and PSEP is weak, these data suggest an increased PESP concentration in the urine sample is correlated with the severity of symptoms and the advanced stages of CP/CPPS.

We have conducted a power analysis by the $G$ power software to assess the sample size. Power $(1-\beta)=0.99$, which means the power of the test is well, and the sample size is enough to have valid results (Figure 1).

\section{Discussion}

CP/CPPS is caused by the interaction between immune, neurological and endocrine systems and psychological factors (22). The theories behind the disease include stress-induced dysfunction of the hypothalamic-pituitaryadrenal axis (23), abnormal adrenal cortex hormones (endocrine) (24), neurogenic inflammation (25) and muscle fascia pain syndrome (26). In the latter two categories, local nervous system disorders are caused by past trauma, infection or anxious personality. Chronic unconscious tightening of the pelvis (regulated by the release of substances from nerve cells, such as substance $\mathrm{P}$ can also lead to inflammation. The prostate and other parts of the urogenital tract: bladder, urethra, testis can also be inflamed by long-term activation of mast cells at the ends of the pelvic nerves. Similar stress-induced genitourinary inflammation has been found in experiments of other mammals (27). However, there is no correlation between the histological examination of prostatitis and the chronic prostatitis symptom index of the NIH-CPSI (28).

$\mathrm{CP} / \mathrm{CPPS}$ is a frustrating clinical condition for both practitioners and patients. In developing countries, the situation is worse because often CP/CPPS is not correctly diagnosed using a combination of multiple methodologies, including NIH-CPSI, EPS, 2 - and 4 cup tests, and so on (29). Instead, clinician experience sometimes dictates the treatment of self-described abdominal and urinary discomfort with a frequent prescription of antibiotics to observe treatment response to the anticipated CP/CPPS.

At present, there is no uniform standard and "gold standard" for the diagnosis of CP/CPPS, and laboratory diagnosis lacks widely accepted biomarkers, and the methodology that can be used in clinical research is very limited. There is no doubt that CP changes the local microenvironment of the prostate, and the corresponding biomarker changes will inevitably occur. Quick et al. (30) found CCL-2 and CCL-3 of CP/CPPS patients has a key inflammatory mediator effect. The results of Paulis et al. (31) showed that IL-6 in EPS of CP/CPPS patients was significantly higher than that in healthy controls, and its increased level was related to CP/CPPS symptom score (NIH-CPSI). Hochreiter et al. (32) found that IL-8 in EPS of type IIIA patients was significantly higher than type IIIB group and the control group, it is believed that IL-8 can be used to distinguish type IIIA and IIIB CP/CPPS. Miller et al. (33) measured NGF, IL-6, IL-8, and IL-10 in semen of CP/CPPS patients, and found that the level of NGF in semen was related to the severity of pain. These findings are consistent with the findings of Watanabe et al. (34) that the NGF in EPS is related to the NIH-CPSI pain score, and the level of NGF is significantly reduced after successful treatment. As a new member of the costimulatory molecule family, B7-H3 plays an important role in regulating the immune system. Wei et al. (35) reported that the B7-H3 in EPS of the control group was significantly higher than other subtypes group, and the B7-H3 level of CP/CPPS III $\mathrm{B}$ patients was higher than that of CP /CPPS IIIA patients. 


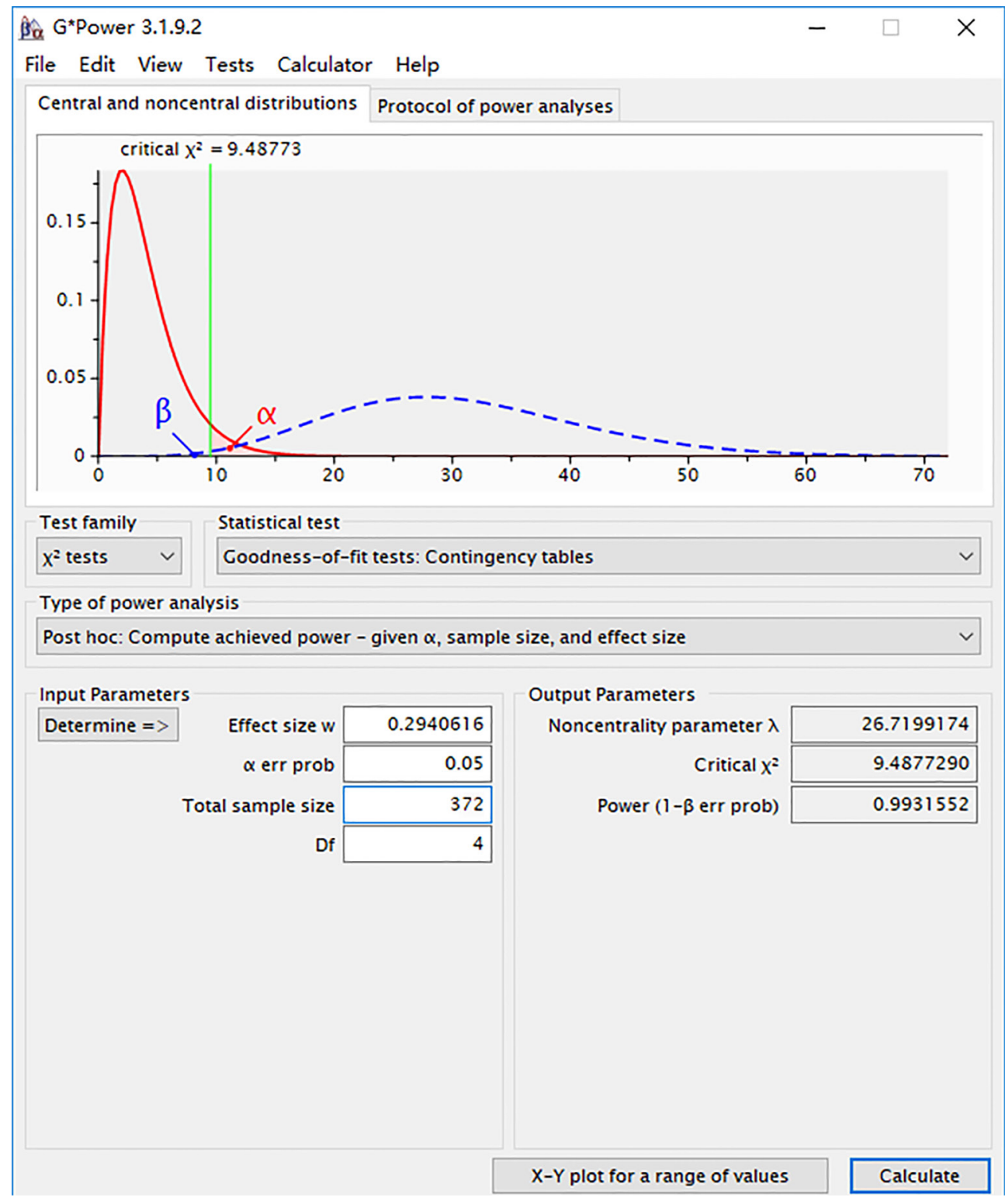

Figure 1 The power analysis by the G power software.

Traditional routine testing methods, including EPS and urine routine diagnostic examination are challenged. It has limited significance for clinical guidance. Therefore, we need to find a new biomarker to guide the diagnosis and treatment of CP/CPPS.

The available PSEP-ELISA assay as a simple, objective and non-invasive urine test supplies the clinician as a biological surrogate to aid in the diagnosis of CP/CPPS. In the past couple of years, the PSEP test became adopted in hospitals and clinics in China and received some positive responses. However, the correlation of urine PSEP level with EPS and/or NIH-CPSI was only described in meetings and conferences anecdotally. Therefore, the current study is the first to confirm that the PSEP level is associated with the increasing WBC counts and NIH-CPSI scores. Thus, an increasing PSEP level in the urine can be a sign of the severity of CP/CPPS and may guide the best treatment plan. However, it is a pilot study that only elaborates on the correlation of urine PSEP level with EPS and/or NIHCPSI. Therefore, further study should focus on the cut- 
off point of PSEP to distinguish between IIIa and IIIb categories avoiding prostatic massage.

During the chronic prostatic process, leukocytes exudate and swarm to the inflammation region; leukocytes engulf lecithin, causing lecithin bodies to decrease.

In our current study, we do not find a strong correlation between urine PSEP level and density of EPS-lecithin corpuscles. We noticed this result and had extensive discussions with other clinicians. We believe that further study may be needed to test this relationship more closely in a study with a larger sample size.

There is intense research ongoing to find better and more practical biomarkers for CP/CPPS. Studies have shown that inflammatory cytokines in seminal plasma of CP/CPPS patients are increased significantly, including IL1, IL-6, IL-8, IL-10, and TNF-a (36). Polymorph nuclear (PMN) elastase in EPS was significantly higher in IIIa compared to IIIb $(37,38)$. Other pathogens than bacteria, including chlamydia, are associated with increased WBC counts and pain severity in men with CP/CPPS. Perhaps the most thorough survey of the protein biomarkers came from mass spectrometry of seminal plasma proteomes of prostatitis patients (39). This study identified 418 proteins associated with prostatitis versus 280 present in healthy individuals with 1,662 proteins present in both populations. While these are encouraging steps towards the development of vial biomarkers for CP/CPPS, they are derived from EPS or require sophisticated equipment to perform analysis. Therefore, they are not of practical value for general clinical application at this moment.

While PSEP-ELISA assay is simple to perform on voided urine, much still is to be learned. For example, it would be essential to confirm our study by more independent hospitals and clinics around the world for different ethnic backgrounds. There are reports that in some regions, pathogens may be more closely related to CP/CPPS. Also, the mechanism of PSEP involvement in CP/CPPS is entirely unknown. It would be essential to understand why PSEP is elevated in CP/CPPS and whether it is causative, or it is a mere biomarker surrogate. The understanding of PSEP biology would also be necessary for drug development. For example, many animal models are currently being used to investigate the etiology and drug response of experimental prostatitis in animal models (40). PSEP may be used to monitor the disease course and drug treatment outcomes. There are still some shortcomings in this study. There is no NIH classification of CP. The correlation between PSEP in urine of CP patients with different types and WBC, SPL counts in EPS, and NIHCPSI score needs further study. We are preparing to carry out a multidisciplinary, multi-center, prospective, largesample clinical study of evidence-based medicine. Moreover, $\mathrm{CP} / \mathrm{CPPS}$ is often clinically associated with symptoms of urinary dysfunction. Currently, research on PSEP only confirms that it is related to the severity of symptoms, but cannot locate the cause. It may be necessary to combine UDS examinations, which is more conducive to accurate treatment of patients. UDS examination can identify specific types of lower urinary tract dysfunction in patients with CP/CPPS (41), and then take targeted treatment.

\section{Conclusions}

Our study proved a correlation between the increase of PSEP level and NIH-CPSI scores. In the meantime, the correlation was found between the PSEP level and EPS indexes. These findings highlight the potential of PSEP is a practical indicator of the symptomatic progression of CP/CPPS. Applications of PSEP assay may guide drug discovery and lead to better treatment to improve the patient's quality of life.

\section{Acknowledgments}

Funding: This work was supported by the Jiangsu Province Social and Development Project (No. BE2017724) and the Special Project of Family Planning in Military (No. 17JS013).

\section{Footnote}

Reporting Checklist: The authors have completed the MDAR reporting checklist. Available at http://dx.doi.org/10.21037/ tau-20-1268

Data Sharing Statement: Available at http://dx.doi. org/10.21037/tau-20-1268

Conflicts of Interest: All authors have completed the ICMJE uniform disclosure form (available at http://dx.doi. org/10.21037/tau-20-1268). The authors have no conflicts of interest to declare.

Ethical Statement: The authors are accountable for all 
aspects of the work in ensuring that questions related to the accuracy or integrity of any part of the work are appropriately investigated and resolved. The Institutional Research Ethics Committee approved all research analysis. The written informed consent was retrieved from all individuals, and case-report-forms (CRF) were administered during outpatient visits to collect the information of age, routine urinary test, EPS and NIH-CPSI, etc. All procedures performed in this study involving human participants were in accordance with the Declaration of Helsinki (as revised in 2013).

Open Access Statement: This is an Open Access article distributed in accordance with the Creative Commons Attribution-NonCommercial-NoDerivs 4.0 International License (CC BY-NC-ND 4.0), which permits the noncommercial replication and distribution of the article with the strict proviso that no changes or edits are made and the original work is properly cited (including links to both the formal publication through the relevant DOI and the license). See: https://creativecommons.org/licenses/by-nc-nd/4.0/.

\section{References}

1. Collins MM, Stafford RS, O'Leary MP, et al. How common is prostatitis? A national survey of physician visits. J Urol 1998;159:1224-8.

2. Clemens JQ, Meenan RT, O'Keeffe Rosetti MC, et al. Prevalence of and risk factors for prostatitis: population based assessment using physician assigned diagnoses. J Urol 2007;178:1333-7.

3. Qin $\mathrm{Z}, \mathrm{Wu} \mathrm{J}, \mathrm{Xu} \mathrm{C}$, et al. Long-term effects of acupuncture for chronic prostatitis/chronic pelvic pain syndrome: systematic review and single-arm meta-analyses. Ann Transl Med 2019;7:113.

4. Weidner W, Schiefer HG, Krauss H, et al. Chronic prostatitis: a thorough search for etiologically involved microorganisms in 1,461 patients. Infection 1991;19:S119-25.

5. Schaeffer AJ. Chronic prostatitis and the chronic pelvic pain syndrome. N Engl J Med 2006;355:1690-8.

6. Breser María L, Salazar Florencia C, Rivero Viginia E, et al. Immunological Mechanisms Underlying Chronic Pelvic Pain and Prostate Inflammation in Chronic Pelvic Pain Syndrome. Front Immunol 2017;8:898.

7. Zhang JZ, Liang CZ, Shang XJ, et al. Chronic Prostatitis/ Chronic Pelvic Pain Syndrome: A Disease or Symptom? Current Perspectives on Diagnosis, Treatment, and
Prognosis. Am J Mens Health 2020;14:1557988320903200.

8. Nickel JC, Downey J, Hunter D. Prevalence of prostatitislike symptoms in a population-based study using the National Institutes of Health chronic prostatitis symptom index. J Urol 2001;165:842-5.

9. Tomas D, Kruslin B, Rogatsch H, et al. Different types of atrophy in the prostate with and without adenocarcinoma. Eur Urol 2007;51:98-103.

10. Hessvik NP, Llorente A. Current knowledge on exosome biogenesis and release. Cell Mol Life Sci 2018;75:193-208.

11. Aalberts M, Sostaric E, Wubbolts R, et al. Spermatozoa recruit prostasomes in response to capacitation induction. Biochim Biophys Acta 2013;1834:2326-35.

12. Brody I, Ronquist G, Gottfries A. Ultrastructural localization of the prostasome-an organelle in human seminal plasma. UPS J Med Sci 1983;88:63-80.

13. Carlsson L, Påhlson C, Bergquist M, et al. Antibacterial activity of human prostasomes. Prostate 2000;44:279-86.

14. Skibinski G, Kelly RW, Harkiss D. Immunosuppression by human seminal plasma- extracellular organelles (prostasomes) modulate activity of phagocytic cells. Am J Reprod Immunol 1992;28:97-103.

15. Saez F, Motta C, Boucher D, et al. Antioxidant capacity of prostasomes in human semen. Mol Hum Reprod 1998;4:667-72.

16. Saez F, Motta C, Boucher D, et al. Prostasomes inhibit the NADPH oxidase activity of human neutrophils. Mol Hum Reprod 2000;6:883-91.

17. Poliakov A, Spilman M, Dokland T, et al. Structural heterogeneity and protein composition of exosomelike vesicles (prostasomes) in human semen. Prostate 2009;69:159-67.

18. Lu Q, Zhang J, Allison R, et al. Identification of extracellular delta-catenin accumulation for prostate cancer detection. Prostate 2009;69:411-8.

19. Minelli A, Ronquist G, Carlsson L, et al. Antiprostasome antibody in benign and malignant prostate disease. Anticancer Res 2005;25:4399-402.

20. Zeng Y, Zhang J, Chen YH. Establishment of ELISA detection method for prostatic exosomal protein and its primary evaluation. Journal of Clinical Medicine \& Pharmacy 2015;32:885-8.

21. Propert KJ, Litwin MS, Wang Y, et al. Responsiveness of the National Institutes of Health Chronic Prostatitis Symptom Index (NIH-CPSI). Qual Life Res 2006;15:299-305.

22. Pontari MA, Ruggieri MR. Mechanisms in prostatitis/ chronic pelvic pain syndrome. J Urol 2008;179:S61-7. 
23. Anderson RU, Orenberg EK, Chan CA, et al. Psychometric Profiles and Hypothalamic-PituitaryAdrenal Axis Function in Men With Chronic Prostatitis/ Chronic Pelvic Pain Syndrome. J Urol 2008;179:956-60.

24. Dimitrakov J, Joffe HV, Soldin SJ, et al. Adrenocortical Hormone Abnormalities in Men with Chronic Prostatitis/ Chronic Pelvic Pain Syndrome. Urology 2008;71:261-6.

25. Sant GR, Kempuraj D, Marchand JE, et al. The mast cell in interstitial cystitis: role in pathophysiology and pathogenesis. Urology 2007;69:34-40.

26. Anderson RU, Wise D, Sawyer T, et al. Integration of myofascial trigger point release and paradoxical relaxation training treatment of chronic pelvic pain in men. J Urol 2005;174:155-60.

27. Alexacos N, Pang X, Boucher W, et al. Neurotensin mediates rat bladder mast cell degranulation triggered by acute psychological stress. Urology 1999;53:1035-40.

28. Nickel JC, Roehrborn CG, O'leary MP, et al. Examination of the relationship between symptoms of prostatitis and histological inflammation: baseline data from the REDUCE chemoprevention trial. J Urol 2007;178:896-900.

29. Na YQ, Ye ZQ, Sun YH, et al. Manual of diagnosis and treatment of diseases in Department of Urology in China. Pmph 2014;568-9.

30. Quick ML, Mukherjee S, Rudick CN, et al. CCL2 and CCL-3 are essential mediators of pelvic pain in experimental autoimmune prostatitis. Am J Physiol Regul Integr Comp Physiol 2012;303:R580-9.

31. Paulis G, Conti E, Voliani S, et al. Evaluation of the cytokines in genital secretions of patients with chronic prostatitis. Arch Ital Urol Androl 2003;75:179-86.

32. Hochreiter WW, Nadler RB, Koch AE, et al. Evaluation of the cytokines interleukin 8 and epithelial neutrophil activating peptide 78 as indicators of inflammation in prostatic secretions. Urology 2000;56:1025-9.

Cite this article as: Liang $\mathrm{W}, \mathrm{Wu} Z$, Zhang $\mathrm{G}$, Chen $\mathrm{W}, \mathrm{Hu}$ X, Yang J, Meng J, Zeng Y, Li H, Shang X. A urine-based biomarker for chronic prostatitis/chronic pelvic pain syndrome: a retrospective multi-center study. Transl Androl Urol 2020;9(5):2218-2226. doi:10.21037/tau-20-1268
33. Miller LJ, Fischer KA, Goralnick SJ, et al. Nerve growth factor and chronic prostatitis/chronic pelvic pain syndrome. Urology 2002;59:603-8.

34. Watanabe T, Inoue $M$, Sasaki K, et al. Nerve growth factor level in the prostatic fluid of patients with chronic prostatitis/chronic pelvic pain syndrome is correlated with symptom severity and response to treatment. BJU Int 2011;108:248-51.

35. Wei X, Zhang G, Yuan H, et al. Detection and quantitation of soluble B7-H3 in expressed prostatic secretions: A novel marker in patients with chronic prostatitis. J Urol 2011;185:532-7.

36. Penna G, Mondaini N, Amuchastegui S, et al. Seminal plasma cytokines and chemokines in prostate inflammation: interleukin 8 as a predictive biomarker in chronic prostatitis/chronic pelvic pain syndrome and benign prostatic hyperplasia. Eur Urol 2007;51:524-33.

37. Aghazarian A, Plas E, Stancik I, et al. New method for differentiating chronic prostatitis/chronic pelvic pain syndrome IIIA from IIIB involving seminal macrophages and monocytes. Urology 2011;78:918-23.

38. Zhu J, Yang C, Dong Z, et al. The value of neutrophil elastase in diagnosis of type III prostatitis. Urol J 2014;11:1666-72.

39. Kagedan D, Lecker I, Batruch I, et al. Characterization of the seminal plasma proteome in men with prostatitis by mass spectrometry. Clin Proteomics 2012;9:2.

40. Vykhovanets EV, Resnick MI, MacLennan GT, et al. Experimental rodent models of prostatitis: limitations and potential. Prostate Cancer Prostatic Dis 2007;10:15-29.

41. Xu SF, Ku AB, Cao P, et al. Urodynamic study of chronic prostatitis/chronic pelvic pain syndrome and its clinical significance. J Clin Sur 2020;28:270-2.

(English Language Editor: J. Chapnick) 